Canadian

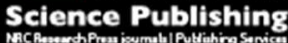

Canadian Journal of Zoology Revue canadienne de zoologie

\title{
Testosterone-related and seasonal changes in sebaceous glands in the back skin of adult male brown bears (Ursus arctos)
}

\begin{tabular}{|c|c|}
\hline Journal: & Canadian Journal of Zoology \\
\hline Manuscript ID & cjz-2017-0028.R2 \\
\hline Manuscript Type: & Article \\
\hline Date Submitted by the Author: & 06-Sep-2017 \\
\hline Complete List of Authors: & $\begin{array}{l}\text { Tomiyasu, Jumpei ; Obihiro University of Agriculture and Veterinary } \\
\text { Medicine, Laboratory of Theriogenology, Department of Applied Veterinary } \\
\text { Medicine; Gifu University, United Graduate School of Veterinary Sciences } \\
\text { Yanagawa, Yojiro; Hokkaido University, Laboratory of Theriogenology, } \\
\text { Department of Veterinary Clinical Sciences } \\
\text { Sato, Yoshikazu; Rakuno Gakuen University, Laboratory of Wildlife Ecology, } \\
\text { Department of Environmental Symbiotic Science, College of Agriculture, } \\
\text { Food and Environmental Sciences } \\
\text { Shimozuru, Michito; Hokkaido University, Graduate School of Veterinary } \\
\text { Medicine } \\
\text { Nagano, Masashi; Hokkaido University, Laboratory of Theriogenology, } \\
\text { Department of Veterinary Clinical Sciences } \\
\text { Sasaki, Motoki; Obihiro University of Agriculture and Veterinary Medicine, } \\
\text { Laboratory of Veterinary Anatomy, Department of Basic Veterinary Science } \\
\text { Sakamoto, Hideyuki; Noboribetsu Bear Park } \\
\text { Matsumoto, Naoya; Noboribetsu Bear Park } \\
\text { Kobayashi, Kohei; EnVision Conservation Office } \\
\text { Kayano, Mitsunori; Obihiro University of Agriculture and Veterinary } \\
\text { Medicine, Research Center for Global Agromedicine } \\
\text { Haneda, Shingo; Obihiro University of Agriculture and Veterinary Medicine, } \\
\text { Laboratory of Theriogenology, Department of Applied Veterinary Medicine } \\
\text { Matsui, Motozumi; Obihiro University of Agriculture and Veterinary } \\
\text { Medicine, Laboratory of Theriogenology, Department of Applied Veterinary } \\
\text { Medicine; Gifu University, United Graduate School of Veterinary Sciences }\end{array}$ \\
\hline Keyword: & $\begin{array}{l}\text { brown bear, sebaceous gland, testosterone, tree rubbing, back skin, scent } \\
\text { gland, }<i>\text { Ursus arctos }</ i>\end{array}$ \\
\hline
\end{tabular}


Testosterone-related and seasonal changes in sebaceous glands in the back skin of adult male brown bears (Ursus arctos)

Running Header: Sebaceous glands in back skin of male brown bears

J. Tomiyasu ${ }^{1,2}$, Y. Yanagawa ${ }^{3}$, Y. Sato ${ }^{4}$, M. Shimozuru ${ }^{5}$, M. Nagano ${ }^{3}$, M. Sasaki ${ }^{2,6}$, H. Sakamoto ${ }^{7}$, N. Matsumoto $^{7}$, K. Kobayashi ${ }^{8}$, M. Kayano ${ }^{9}$, S. Haneda ${ }^{1}$ and M. Matsui ${ }^{1,2 *}$

${ }^{1}$ Laboratory of Theriogenology, Department of Applied Veterinary Medicine, Obihiro University of Agriculture and Veterinary Medicine, Obihiro, Hokkaido 080-8555, Japan

${ }^{2}$ United Graduate School of Veterinary Sciences, Gifu University, Gifu, Gifu 501-1193, Japan

${ }^{3}$ Laboratory of Theriogenology, Department of Veterinary Clinical Sciences, Graduate School of Veterinary Medicine, Hokkaido University, Sapporo, Hokkaido 060-0818, Japan

${ }^{4}$ Laboratory of Wildlife Ecology, Department of Environmental Symbiotic Science, College of Agriculture, Food and Environmental Sciences, Rakuno Gakuen University, Ebetsu, Hokkaido 069-0836, Japan

${ }^{5}$ Laboratory of Wildlife Biology and Medicine, Department of Environmental Veterinary Science Graduate School of Veterinary Medicine, Hokkaido University, Sapporo, Hokkaido 060-0818, Japan ${ }^{6}$ Laboratory of Veterinary Anatomy, Department of Basic Veterinary Science, Obihiro University of Agriculture and Veterinary Medicine, Obihiro, Hokkaido 080-8555, Japan

${ }^{7}$ Noboribetsu Bear Park, Noboribetsu, Hokkaido 059-0551, Japan

${ }^{8}$ EnVision Conservation Office, 5-2, Kita 9, Nishi 4, Kita-ku, Sapporo, Hokkaido 060-0809, Japan

${ }^{9}$ Research Center for Global Agromedicine, Obihiro University of Agriculture and Veterinary Medicine, Obihiro, Hokkaido 080-8555, Japan

J. Tomiyasu, j.tomiyasu@gmail.com; Y. Yanagawa,yoji-y@vetmed.hokudai.ac.jp;

Y. Sato, ysato@rakuno.ac.jp; M. Shimozuru, shimozuru@vetmed.hokudai.ac.jp;

M. Nagano,mnaga@vetmed.hokudai.ac.jp; M. Sasaki, sasakim@obihiro.ac.jp;

H. Sakamoto, sakamoto@bearpark.jp; N. Matsumoto, naoyamatsumotoster@gmail.com;

K. Kobayashi, k.kobayashi731@gmail.com; M. Kayano, kayano@obihiro.ac.jp;

S. Haneda, haneda@obihiro.ac.jp; M. Matsui*, mmatsui@obihiro.ac.jp

*Corresponding author

Motozumi Matsui, Tel: +81-155-49-5382, Fax: +81-155-49-5384

Email:mmatsui@obihiro.ac.jp

Postal address: Nishi-2-11, Inada-cho, Obihiro, Hokkaido, 080-8555, Japan 


\section{Testosterone-related and seasonal changes in sebaceous glands in the back skin of adult male brown bears (Ursus arctos)}

Jumpei Tomiyasu, Yojiro Yanagawa, Yoshikazu Sato, Michito Shimozuru, Masashi Nagano, Motoki Sasaki, Hideyuki Sakamoto, Naoya Matsumoto, Kohei Kobayashi, Mitsunori Kayano, Shingo Haneda and Motozumi Matsui

\section{Abstract}

Adult male brown bears (Ursus arctos; Linnaeus, 1758) display tree-marking behavior to chemically signalize the dominance throughout the non-denning period, and this behavior peaks during breeding season. Within the scent-marking sequence, back rub is one of a core marking postures. The present study investigated 1) seasonal changes in sebaceous glands in the back skin of brown bears and 2) the relationship between those changes and testosterone levels. Back skin tissue samples and blood were collected from captive adult intact and castrated males during pre-breeding, transitional, breeding and post-breeding seasons, which were concurrent with back skin observations. In intact males, during the transitional and breeding seasons, an oily secretion from the back skin was observed along with enlarged sebaceous glands. The plasma testosterone concentrations during the transitional and breeding seasons were increased compared with the pre- and post-breeding seasons. Secretions and enlarged sebaceous glands were not found in castrated males, and the plasma testosterone concentrations remained at baseline levels. Oily secretions of the back skin glands that appear more abundant during breeding season are rubbed against trees. Changes in size and volume of sebaceous glands, and thus their secreting capacity, are likely testosterone-regulated.

Keywords: brown bear, sebaceous gland, testosterone, tree-rubbing, back skin, scent gland, Ursus arctos 


\section{Introduction}

Olfactory communication is the primary form of communication between individuals in many mammalian species, and it represents a key source of information (Gorman and Trowbridge 1989; Müller-Schwarze 2006). Animals use feces, urine, and glandular secretions from skin to communicate chemically (Müller-Schwarze 2006) without the need for direct contact. Odors remain viable for long periods, even in the absence of the producer (Margaret et al. 1980).

Brown bears (Ursus arctos; Linnaeus, 1758) are normally solitary with a large home range (Dahle and Swenson 2003; Støen et al. 2005); therefore, scent is a valuable mechanism of information transmission. Because the mating system of brown bears is polygynous, adult males fight with each other for opportunities to mate with females during the breeding season (May-July; Tsubota et al. 1985; Ishikawa et al. 2003). Young male bears disperse from their natal areas, probably to avoid mating competition with older bears (Zedrosser et al. 2007), and they are likely able to identify sexually mature male brown bears by odors emitted from anal sacs (Rosell et al. 2011; Jojola et al. 2012). Sub-adult male bears might store information derived from adult male odors for future encounters to reduce the cost of conflict (Jojola et al. 2012). Therefore, brown bears seem to use olfactory communication.

Brown bears often rub themselves against trees (Green and Mattson 2003; Karamalidis et al. 2007; Clapham et al. 2014; Sato et al. 2014) placed along travel routes, perhaps as landmarks (Green and Mattson 2003, Clapham et al. 2013). Adult male bears rub against trees more often than other age and sex classes throughout the non-denning period, and 
adult males might utilize chemical signaling to impart dominance information (Clapham et al. 2012). This behavior peaks during the breeding season (Green and Mattson 2003; Clapham et al. 2012, 2014; Sato et al. 2014). A study of the motor activity of sent marking in brown bears (Clapham et al. 2014), identified bipedal back rubbing as a core marking posture. Elevated marking behavior might widely disperse information and increase detectability (Albert et al. 1992), or convey competitive ability (White et al. 2002). We speculated that male brown bears communicate chemically by rubbing the skin on the back against surfaces during the breeding season, and that information derived from this behavior is related to reproduction.

Wild brown bears seem to increase the amount of tree-rubbings during the breeding season (Green and Mattson 2003; Clapham et al. 2012, 2014; Sato et al. 2014). We hypothesized that male brown bears have sebaceous glands in back skin that become enlarged by testosterone during the breeding season. This is because sebaceous gland enlargement depends on testosterone levels in goats Capra hircus (L., 1758) (Iwata et al. 2000; Wakabayashi et al. 2000), golden hamsters Mesocricetus auratus (Waterhouse, 1839) (Vandenbergh 1973), rat-like hamsters Cricetulus triton (de Winton, 1899) (Zhang et al. 2001), and musk rats Ondatra zibethica (L., 1766) (Lu et al. 2014). In order to investigate the characteristic of sebaceous glands in skin from the back of brown bear, the present study aimed 1) to define seasonal changes in sebaceous glands relative to breeding season and: 2) to clarify the relationship between those changes and testosterone levels.

\section{Materials and methods}




\section{Experimental design}

We investigated seasonal changes in sebaceous glands in skin from the backs of bears as follows. We firstly visually assessed the skin on backs of anesthetized captive intact bears and collected skin samples for histological analysis during the following seasons: pre-breeding (February), transitional (April), breeding (May and June; Tsubota et al. 1985; Ishikawa et al. 2003), and post-breeding (August and October), which is classified on the basis of the previous study about testosterone of male brown bears (Tsubota and Kanagawa 1989). To examine whether or not sebaceous gland enlargement is specific to skin on the back during the breeding season, we compared skin sampled from back and rump, because back rubbing is a core posture, whereas rump rubbing is not (Clapham et al. 2014). Moreover, to examine the relationship between those changes in sebaceous glands and testosterone levels, we measured plasma testosterone concentrations and the size of the sebaceous glands in intact and castrated male bears. The presence of androgen receptors was immunohistochemically assessed.

\section{Animals}

We studied 11 captive Hokkaido male brown bears from the Noribetsu Bear Park, Hokkaido, Japan $\left(42^{\circ} \mathrm{N}, 141^{\circ} \mathrm{E}\right.$ : Facility A; five individuals: four intact and one castrated), and Sahoro Bear Mountain, Hokkaido Japan $\left(43^{\circ} \mathrm{N}, 142^{\circ} \mathrm{E}\right.$ : Facility B; six individuals: four intact and two castrated). We used five of seven bears that spent the daytime outdoors in a $700 \mathrm{~m}^{2}$ open concrete space at Facility A. At night, four of them were placed in a $23 \times 2.3$

$\times 2.2 \mathrm{~m}^{3}$ (height $\times$ width $\times$ depth). Three others were placed in a $12 \times 2.3 \times 2.2 \mathrm{~m}^{3}$ (height $\times$ width $\times$ depth). We used six of ten bears which were placed in an open, fenced 
mountainous area of $150,000 \mathrm{~m}^{2}$ during the daytime at Facility B. The vegetation in this area was similar to that in wild-bear habitats. And, they were separated in one space, respectively at night in a $2.2 \times 2.2 \times 2.5 \mathrm{~m}^{3}$ (height $\times$ width $\times$ depth) area. The bears were fed bear pellets (ZOOFOOD bear, Nosan Co., Kanagawa, Japan), concentrated feed formulated for cows (Soyokazenokaori MG, Nippon Formula Food Manufacturing Co., Ltd., Kanagawa Japan), vegetables, and fruits once or twice a day with water ad libitum. All the bears studied herein was born in captivity. Rubbing behavior occurred during the study period. Bears rubbed themselves against walls in the absence of trees in Facility A. Male and female bears were separated in Facility A, but a short distance between pens allowed for olfactory and auditory cognition. Facility B did not house any female bears. All bears were between eight to 25 years of age, and were sexually mature. The bears were exposed to natural photoperiods throughout the year.

Skin examination and tissue collection

Samples were collected from the same individuals at Facility A during February, April, May, June, August, and October, and at Facility B in April, June, and October (Table 1). The bears were repeatedly anesthetized via the intramuscular administration of 2.5-3.5 $\mathrm{mg} / \mathrm{kg}$ of a mixture of zolazepam $\mathrm{HCl}$ and tiletamine $\mathrm{HCl}$ (Zoletil, Virbac, Carros, France) with either $0.03 \mathrm{mg} / \mathrm{kg}$ medetomidine $\mathrm{HCl}$ (Domitor; Orion Corporation Animal Health, Turku, Finland) or $1 \mathrm{mg} / \mathrm{kg}$ xylazine $\mathrm{HCl}$ (Selactar; Bayer Healthcare, Leverkusen, Germany) using blow darts. After sample collection, anesthesia was reversed by the intramuscular administration of $0.03 \mathrm{mg} / \mathrm{kg}$ atipamezole $\mathrm{HCl}$ (Antisedan; Orion Corporation Animal Health). Secretions were verified directly by observation and by 
rubbing skin with a paper towel. Skin samples were obtained from the center of the back skin between the shoulder blades (Fig. 1) using an 8-mm BP-L80K Biopsy Punch (Kai, Ind., Ltd., Gifu, Japan), and all biopsies were obtained at a depth of $25 \mathrm{~mm}$. Skin samples from the rump were also collected in June from four intact bears at Facility B. A section of fur on each bear was shaved, the skin was repeatedly washed with $20 \mathrm{mg} / \mathrm{mL}$ povidone iodine (Isodine; Meiji Seika, Tokyo, Japan) and iso-propanol 50\% (iso-propanol 50\%, Yakuhan, Kitahiroshima, Japan) and then veterinarians collected biopsy specimens. The wound was then sutured with Coated Vicryl ${ }^{\circledR}$ Plus Antibacterial Suture (Ethicon, Somerville, NJ). After completing all experiments, $0.2 \mathrm{mg} / \mathrm{kg}$ meloxicam (Metacam, Boehringer Ingelheim, Germany) and $5 \mathrm{mg} / \mathrm{kg}$ Enrofloxacin (Baytril, Bayer, Leverkusen, Germany) were subcutaneously administered for analgesia and antimicrobial activity, respectively. All procedures were conducted by veterinarians. Pus was not evident at the biopsy sites on the following day. Skin biopsy specimens were fixed in $10 \%$ buffered formalin. Blood samples were collected from the saphenous veins into Venoject® II heparinized Vacutainers ${ }^{\circledR}$ (Terumo, Tokyo, Japan), and the plsma was separated from hematocytes by centrifugation at $2000 \times g$ for $15 \mathrm{~min}$. Plasma samples were stored at $-30{ }^{\circ} \mathrm{C}$ until the assay was performed.

\section{Histological and immunohistochemical analysis}

Fixed skin tissues were dehydrated in serially diluted ethanol, embedded in paraffin (Paraplast Plus; Kendall, Mansfield, MA, USA), cut into 4- $\mu \mathrm{m}$ thick sections and mounted onto Mas-coated slides (S9904, Matsunami Glass Ind., Ltd., Osaka, Japan). To examine the skin glands, deparaffinized sections were stained with hematoxylin and eosin. 
Androgen receptor (AR) expression was immunohistochemically assessed using the avidin-biotin complex (ABC) method (Hsu et al. 1981). Samples of skin collected from intact and castrated back, and intact rump in June were prepared for immunohistological assessment as follows. Deparaffinized sections were incubated in a LAB antigen retrieval solution (Polysciences Inc, Eppelheim, Germany) at $60{ }^{\circ} \mathrm{C}$ for $60 \mathrm{~min}$, and then soaked in $0.3 \% \mathrm{H}_{2} \mathrm{O}_{2}$ in methanol for $10 \mathrm{~min}$ to inactivate the endogenic peroxidase. Nonspecific binding was blocked by incubating the sections with a 1:50 dilution of S-1000 normal goat serum (Vector Laboratories, Inc., CA, USA) for $30 \mathrm{~min}$ at room temperature. The slides were then incubated at $4{ }^{\circ} \mathrm{C}$ overnight with a 1:50 dilution of $\mathrm{N}-20$ rabbit polyclonal anti-androgen receptor antibody (Santa Cruz Biotechnology, Inc., TX, USA), followed by $30 \mathrm{~min}$ at room temperature with 1:200 dilution of BA-1000 goat biotinylated anti-rabbit IgG (Vector Laboratories, Inc.). The samples were exposed to the $\mathrm{ABC}$ reagent in PK-6100, Vectastain Elite ABC Kit (Vector Laboratories) at room temperature for 30 min and then primary antibody binding sites were visualized by soaking the sections in $0.02 \%$ 3,3'-diaminobenzidine $\mathrm{HCl}$ and $0.006 \% \mathrm{H}_{2} \mathrm{O}_{2}$ in Tris- $\mathrm{HCl}$ buffer. The sections on slides were finally dehydrated and coverslipped. The negative controls were normal rabbit serum or $0.01 \mathrm{M}$ phosphate buffered saline instead of the primary antibody.

\section{Sebaceous glands morphology or parameters}

Columnar skin biopsy specimens were cut parallel to the long axis at $200-\mu \mathrm{m}$ intervals. Areas of sebaceous glands within $6 \times 10-\mathrm{mm}^{2}$ rectangles on 10 slides were measured using image $\mathrm{J}$ software and summed. This parameter is referred to herein as the "sebaceous glands area" and it served as an index of change in the size of sebaceous glands. 


\section{Hormone assay}

Plasma testosterone concentrations were measured with an enzyme-immunoassay (EIA) using anti-testosterone serum (COSMO FKA 102E, Cosmo Bio Co., Ltd., Inc., Tokyo, Japan) and horseradish peroxidase-labeled testosterone (COSMO FKA 101, Cosmo Bio Co., Ltd., Inc.). The EIA procedure was conducted as previously described (Miyamoto et al., 1992). Testosterone was extracted from the plasma sample with diethyl ether and was subsequently measured, and the extraction efficiency was $91.5 \%$. The standard curve ranged from 0.025 to $25 \mathrm{ng} / \mathrm{mL}$, and $50 \%$ of the effective dose of the assay was 0.462 $\mathrm{ng} / \mathrm{mL}$. The average intra- and inter-assay coefficient of variation values were $7.8 \%$ and $8.1 \%$, respectively.

Statistical analysis

Nonparametric analyses were performed, since the sample size was too small to assume normal distribution. A Kruskal-Wallis test was performed to detect significant differences in sebaceous gland size indices and plasma testosterone concentrations between intact males assigned to different seasonal groups, and a Steel-Dwass test was performed to verify the group pairs that showed significant differences. Spearman's rank correlation test was performed to investigate the correlation between the sebaceous gland size indices and the plasma testosterone concentrations in intact males. The sebaceous glands size indices and the plasma testosterone concentrations between intact and castrated males were compared using the Wilcoxon rank sum test for each season. For samples collected in June, the sebaceous gland size indices between the back and rump skins of intact males were compared using the Wilcoxon rank sum test. All data were statistically analyzed using R 
software (R Development Core Team, 2015), and a $P$-value of $<0.05$ and $<0.1$ indicated a significant differences and tendencies, respectively. Data are presented as the median (range).

\section{Results}

\section{Skin observations}

Abundant sebum secretion (Fig. 1) was observed on the back skin during the transitional $(n=6 / 6)$ and breeding $(n=11 / 12)$ seasons. The secretion was observed at the midline of the back skin from the first cervical vertebra to the second lumbar vertebra (Fig. 1). The secretion had a very strong sweet odor, and the color was brown or colorless. Oily secretion was not evident beyond this specific area of back skin. During the pre- $(n=4)$ and post-breeding $(n=12)$ seasons, no secretions from back skins of males were detected from back skins of males. Throughout the experiment, no secretions were found on the back skin of castrated males ( $n=3$, all seasons).

\section{Seasonal changes in sebaceous skin glands and plasma testosterone concentrations}

Figure 2 shows the histological findings of back skin samples from intact males collected during the study period. Enlarged sebaceous glands were situated next to follicles during the transitional $(n=6 / 6)$ and breeding $(n=11 / 12)$ seasons, with the exception of one male sampled in June that had shriveled sebaceous glands that did not secrete sebum. The sebaceous glands in back skins of intact males shriveled during the pre- $(n=4)$ and post-breeding $(n=12)$ seasons. In castrated males, sebaceous glands did not become enlarged throughout the experimental period ( $n=3$, all seasons). Changes in the index of 
sebaceous gland size in intact males during the pre-breeding $(n=4)$, transitional $(n=6)$, breeding $(n=12)$ and post-breeding $(n=12)$ are shown in Fig. 3. Indices of sebaceous gland size tended to be higher during the transitional and breeding seasons than during the pre-breeding season $(P=0.05$ and $P=0.07$, respectively), and the indices were significantly higher than that of the post-breeding season $(P<0.05$ and $P<0.05$, respectively). The sebaceous gland indices were significantly higher in intact males than in castrated males during the transitional and breeding seasons (transitional, $P<0.05$; intact, 91.5 [45.4 - 148.8]; castrated, $3.0[0.9-3.5]$ and breeding, $P<0.05$; Intact, 100.2 [5.6-159.1]; castrated 9.7 [3.1 - 10.5]; intact, $n=12$, castrated, $n=3)$. Indices of sebaceous gland size in intact males were larger during June from back skin than the rump skin $(n=$ 4; back, 71.1 [38.6 - 148.5]; rump $1.2[0.4-2.4] ; P<0.05)$. The present study indicated that the plasma testosterone concentrations of the intact male bears were seasonally influenced (pre-breeding: $n=4$; transitional: $n=6$, breeding: $n=12$; post-breeding: $n=12$;

Fig. 3). Moreover, the plasma concentration of testosterone tended to be higher during the transitional season than the pre-breeding season $(P=0.05)$, and significantly higher than during the post-breeding season $(P<0.05)$. The plasma testosterone concentrations were significantly higher during the breeding season than during the pre- and post-breeding seasons (both $P<0.05$ ). Plasma testosterone concentrations were significantly higher in intact males than in castrated males during the transitional and breeding seasons (transitional, $P<0.05$; intact, 7.38 [2.20 - 33.85]; castrated, 0.08 [0.05 - 0.23]; breeding, $P$ $<0.05$; intact, 7.39 [1.20 - 46.25], castrated 0.16 [0.13 - 0.17], respectively; intact: $n=12$, castrated: $n=3$; Fig. 3). Significant positive correlations were observed between plasma 
testosterone concentrations and the index of sebaceous gland size in intact males $(P<0.05$; $r=0.78$; pre-breeding: $n=4$; transitional: $n=6$, breeding: $n=12$; post-breeding: $n=12$; Fig. 4).

Immunohistochemistry of AR

Staining for AR in the nuclei of sebaceous gland cells was positive in the back skin samples collected from all eight intact and three castrated bears in June (Fig. 5). Staining for AR was also positive in the intact male with shriveled sebaceous glands in the back skin. Furthermore, the sebaceous glands in the rump skin $(n=4 / 4)$ samples of four males exhibited positive staining for AR.

\section{Discussion}

To our knowledge, this is the first study to show enlarged sebaceous glands and oily skin secretions in the backs of male brown bears during the breeding season. These findings supported our hypothesis that bipedal back rubbing is associated with chemical communication in reproduction.

Glandular structures in mammalian skin are composed of sebaceous, apocrine and eccrine glands. Secretions from skin glands are closely associated with olfactory communication (Müller-Schwarze 2006). The structures of these glands might become more complex structures in specific skin areas (Müller-Schwarze et al. 1977). The location of mammalian scent glands is often linked to motor patterns of scent marking (Stoeckelhuber et al. 2000; Rosell et al. 2011). In fact, pedal scent glands (prominent apocrine and sebaceous glands) in brown bears might be associated with pedal marking 
behavior (Sergiel et al. 2017). Bipedal back rubbing is a core marking posture of bears. The sebaceous glands in the withers of a male brown bear were enlarged compared with those of breast in autumn (Sokolov 1982). We also found an oily secretion and enlarged sebaceous glands in back skin during the breeding season. Thus, back sebaceous glands seemed to be involved in back rubbing. We did not analyze the secretion and secretory status of apocrine glands. Further study is needed to clarify the contribution of apocrine glands to the oily secretion.

The finding that plasma testosterone concentrations were increased in all intact male bears during transitional and breeding seasons is consistent with those of a previous study (Tsubota and Kanagawa 1989). In rabbits Oryctolagus cuniculus (L., 1758), rats Rattus norvegicus (Berkenhout, 1769), guinea pigs Cavia porcellus (L., 1758), and golden hamsters, the enlargement of sebaceous glands is positively correlated with plasma testosterone concentrations (Ebling 1977). Yet, paracloacal glands of marsupials are thought to be a form of scent glands, and Helder-José and Freymüller (1995) suggested that they are modified sebaceous glands. Bradley and Stoddart (1993) associated the enlargement of these glands with high levels of testosterone in the breeding season in the Australian marsupial Petaurus breviceps (Waterhouse, 1838). The neotropical marsupial Metachirus nudicaudatus (É. Geoffroy Saint-Hilaire, 1803) has a pair of yellow and brown paracloacal glands on both right and left sides of the cloaca. Their holocrine secretory epithelium is modulated in a complex way by estrogens in females (Helder-José et al. 2014) and by testosterone in males (Helder-José et al. 2016). Our results corroborated the influence of testosterone upon the sebaceous glands in the back skin of the male brown 
bears. Although sebaceous glands do not enlarge in castrated goats, testosterone supplementation increased the size of these glands (Iwata et al. 2000; Wakabayashi et al. 2000). These results indicated that testosterone regulates the size of sebaceous glands. The present study identified a significant positive correlation between plasma testosterone concentrations and the size index of sebaceous gland in intact male brown bears, and sebaceous glands in the back skin were AR positive. Sebaceous glands in the back skin of three castrated bears shriveled during the transitional and breeding seasons, whereas those in intact males were enlarged. Therefore, testosterone might induce the enlargement of sebaceous glands in the back skin during the transitional and breeding seasons in this species. The regulation of the size of sebaceous gland by testosterone would indicate that back scent glands could convey reproductive information.

The sebaceous glands in the back skins of intact and castrated males stained positively for AR, which also suggests that the size of sebaceous glands in back skin was regulated by testosterone. Testosterone concentrations increased in intact males when the sebaceous glands in back skin became enlarged, but remained at the basal level in castrated males, which explains the shriveled sebaceous glands in back skin. Although sebaceous glands in the rump skin of intact males also stained positively for $A R$, sebaceous glands in the rump did not become enlarged under high plasma testosterone concentrations during the breeding season. The reason why the oily secretion was absent and the sebaceous glands did not become enlarged in rump skin during the breeding season remain unclear. The differential expression of AR and enzymes that are associated with testosterone might have contributed to the differences in the size of sebaceous glands between back and 
rump skin.

Enlarged sebaceous glands were not evident in one intact male during June, even though the serum testosterone level did not differ from those of the other seven intact males. Moreover, the sebaceous glands from this animal specimen stained positive for AR. In Mongolian gerbils (Meriones unguiculatus; Milne-Edwards, 1867), social stress decreased the size of the ventral gland independently of testosterone (Yamaguchi et al. 2005). This bear was housed with other bears that had enlarged sebaceous glands. Body size is thought to be a determinant of male brown bear status (Zedrosser et al. 2007). We postulate that this bear might have been under stress because it weighed less than the others during June. Taken together, social stress might inhibit the enlargement of sebaceous glands in brown bears.

In conclusion, the sebaceous glands in male bears became enlarged concurrently with high plasma testosterone concentrations during the transitional and breeding seasons. Moreover, sebaceous skin glands in the back have AR, so the size of the sebaceous glands in the back skin of this species might be regulated by testosterone. Further investigations, including analyses of secreted components and the effects of the secretion on the behavior of male and female brown bears are required to elucidate the role of these secretions in this species.

\section{Acknowledgements}

The authors thank the staff at Noboribetsu Bear Park (Noboribetsu, Japan), and Sahoro Bear Mountain (Shintoku, Japan) for cooperation during this study. 


\section{Ethical statement}

This experiment was approved by the Animal Experiment Committee of the Obihiro University of Agriculture and Veterinary Medicine, Japan (no. 28-218). 


\section{References}

Albers, A.C. 1992. Constraints on the design communication systems in terrestrial vertebrates. Am. Nat. 139: S62-S89. doi:10.1086/285305.

Bradley, A.J., and Stoddart D.M. 1993. The dorsal paracloacal gland and its relationship with seasonal changes in cutaneous scent gland morphology and plasma androgen in the marsupial sugar glider (Petaurus breviceps; Marsupialia: Petauridae). J. Zool. (Lond.) 229(2): 331-346. doi:10.1111/j.1469-7998.1993.tb02640.x.

Clapham, M., Nevin, O.T., Ramsey, A.D., and Rosell, F. 2012. A hypothetico-deductive approach to assessing the social function of chemical signaling in a non-territorial solitary carnivore. PLoS ONE, 7(4): e35404. doi:10.1371/journal.pone.0035404.

Clapham, M., Nevin, O.T., Ramsey, A.D., and Rosell, F. 2013. The function of strategic tree selectivity in the chemical signaling of brown bears. Anim. Behav. 85: 1351-1357. doi:10.1016/j.anbehav.2013.03.026.

Clapham, M., Nevin, O.T., Ramsey, A.D., and Rosell, F. 2014. Scent-marking investment and motor patterns are affected by the age and sex of wild brown bears. Anim. Behav. 94: 107-116. doi:10.1016/j.anbehav.2014.05.017.

Dahle, B., and Swenson, J.E. 2003. Seasonal range size in relation to reproductive strategies in brown bears Ursus arctos. J. Anim. Ecol. 72(4): 660-667. doi:10.1046/j.1365-2656.2003.00737.x.

Ebling, F.J. 1977. Hormonal control of mammalian skin glands. In Chemical signals in vertebrates. Edited by S.D. Müller and M.M. Mozell. Plenum Press, New York. pp. $17-33$. 
Gorman, M.L., and Trowbridge, B.J. 1989. The role of odor in the social lives of carnivores. In Carnivore behavior, ecology, and evolution. Edited by J.L. Gittleman. Cornell University Press, New York. pp. 57-88.

Green, G.I., and Mattson, D.J. 2003. Tree rubbing by Yellowstone grizzly bears Ursus arctos. Wildl. Biol. 9(1): 1-9.

Helder- José, H., and Freymüller, E. 1995. A morphological and ultrastructural study of the paracloacal (scent) glands of the marsupial Metachirus nudicaudatus Geoffroy, 1803. Acta. Anat. 153(1):31-38. doi:10.1159/000147712. PMID:8560957.

Helder-José, H., Mendes, E.G., Carneiro. N.M., Simões, M.J., and Freymüller, E. 2014. Morphophysiology of the paracloacal (scent) glands in females of the marsupial Metachirus nudicaudatus: action of estrogens. Zoomorphology, 133(2): 237-243. doi:10.1007/s00435-014-0217-8.

Helder-José, H., Simões, M. J., and Freymüller, E. 2016. Testosterone modulation of the male marsupial Metachirus nudicaudatus paracloacal (scent) glands. Zoomorphology, 135(3): 375-385, 2016. doi:10.1007/s00435-016-0314-y.

Hsu, S.M., Raine, L., and Fanger, H. 1981. Use of avidin-biotin-peroxidase complex $(\mathrm{ABC})$ in immunoperoxidase techniques: a comparison between $\mathrm{ABC}$ and unlabeled antibody (PAP) procedures. J. Histochem. Cytochem. 29(4): 577-580. PMID:6166661.

Ishikawa, A., Sakamoto, H., Katagiri, S., and Takahashi, Y. 2003. Changes in sexual behavior and fecal steroid hormone concentrations during the breeding season in 
female Hokkaido brown bears (Ursus arctos yesoensis) under captive condition. J. Vet. Med. Sci. 65(1): 99-102. doi:10.1292/jvms.65.99. PMID: 12576712.

Iwata, E., Wakabayashi, Y., Kakuma, Y., Kikusui, T., Takeuchi, Y., and Mori Y. 2000. Testosterone-dependent primer pheromone production in the sebaceous gland of male goat. Biol. Reprod. 62(3): 806-810. doi.10.1095/biolreprod62.3.806. PMID:10684827.

Jojola, S.M., Rosell, F., Warrington, I., Swenson, J.E., and Zedrossera, A. 2012. Subadult brown bears (Ursus arctos) discriminate between unfamiliar adult male and female anal gland secretion. Mamm. Biol. 77(5): 363-368. doi:10.1016/j.mambio.2012.05.003.

Lu, L., Liu, S., Li, Q., Huang, S., Bao, L., Sheng, X., Han, Y., Watanabe, G., Taya, K., and Weng, Q. 2014. Seasonal expression of androgen receptor in scented gland of muskrat (Ondatra zibethicus). Gen. Comp. Endocrinol. 204: 1-7. doi:10.1016/j.ygcen.2014.04.031. PMID:24818970.

Margaret, E.J., Mills, M.G.L., and Gorman, M.L. 1980. The scent marking behaviour of the brown hyaena brunnea. Afr. Zool. 15(4): 240-248. doi:10.1080/02541858.1980.11447718.

Miyamoto, A., Okuda, K., Schweigert, F.J., and Schams, D. 1992. Effects of basic fibroblast growth factor, transforming growth factor-beta and nerve growth factor on the secretory function of the bovine corpus luteum in vitro. J. Endocrinol. 135(1): 103-114. doi:10.1677/joe.0.1350103. PMID:1431675. 
Müller-Schwarze, D., Quay, W.B., and Brundin, A. 1977. The caudal gland in reindeer (Rangifer tarandus L.): Its behavioral role, histology, and chemistry. J. Chem. Ecol. 3(5): 591-601. doi:10.1007/BF00989079.

Müller-Schwarze, D. 2006. Chemical ecology of vertebrates. Cambridge University Press, Cambridge, U.K.

Rosell, F., Jojola, S.M., Ingdal, K., Lassen, B.A. Swenson, J.E., Arnemo, J.M., and Zedrossera, A. 2011. Brown bears possess anal sacs and secretions may code for sex. J. Zool. (Lond.) 283(2): 143-152. doi:10.1111/j.1469-7998.2010.00754.x.

Sato, Y., Kamiishi, C., Tokaji, T., Mori, M., Koizumi, S., Kobayashi, K., Itoh, T., Sonohara, W., Takada, B.M., and Urata, T. 2014. Selection of rub trees by brown bears (Ursus arctos) in Hokkaido, Japan. Acta Theriol. 59(1): 129-137. doi:10.1007/s13364-013-0143-z.

Sergiel, A., Naves, J., Kujawski, P., Maślak, R., Serwa,E., Ramos, D., Fernández-Gil, A., Revilla, E., Zwijacz-Kozica, T., Zięba, F., Painer, J., and Selva, N. 2017. Histological, chemical and behavioural evidence of pedal communication in brown bears. Sci. Rep. 7:1052. doi:10.1038/s41598-017-01136-1. PMID: 28432322.

Sokolov, V.E. 1982. Mammal skin. University of California Press. Berkeley, C.A.

Støen, O.G., Bellemain, E., Sæbø, S., and Swenson, J.E. 2005. Kin-related spatial structure in brown bears Ursus arctos. Behav. Ecol. Sociobiol. 59(2): 191-197. doi:10.1007/s00265-005-0024-9.

Stoeckelhuber, M., Sliwa, A., and Welsch, U. 2000. Histo-physiology of the scent-marking glands of the penile pad, anal pouch, and the forefoot in the aardwolf (Proteles 
$\begin{array}{llll}\text { cristatus). } & \text { Anat. } & \text { Rec. } & \text { 259(3): }\end{array}$ doi:10.1002/1097-0185(20000701)259:3<312::AID-AR80>3.0.CO;2-X. PMID:10861364.

Tsubota, T., Kanagawa, H., Takahashi, K., Yasue, K and Fukunaga, S. 1985. Observation of sexual behavior under captive conditions in Hokkaido brown bears. Jpn. J. Anim. Reprod. 31(4); 203-210. (in Japanese with English summary). doi:10.1262/jrd1977.31.203.

Tsubota, T., and Kanagawa, H. 1989. Annual changes in serum testosterone levels and spermatogenesis in the Hokkaido brown bear, Ursus arctos yesoensis. J. Mamm. Soc. Jpn. 14(1): 11-17. doi:10.11238/jmammsocjapan1987.14.11.

Vandenbergh, J.G. 1973. Effects of gonadal hormones on the flank gland of the golden hamster. Horm. Res. 4(1): 28-33. doi:10.1159/000178287. PMID:4748463.

Wakabayashi, Y., Iwata, E., Kikusui, T., Takeuchi, Y., and Mori, Y. 2000. Regional differences of pheromone production in the sebaceous glands of castrated goats treated with testosterone. J. Vet. Med. Sci. 62(10): 1067-1072. doi:10.1292/jvms.62.1067. PMID:11073077.

White, A.M., Swaisgood, R.R., and Zhang, H. 2002. The highs and lows of chemical communication in giant pandas (Ailuropoda melanoleuca): effect of scent deposition height on signal discrimination. Behav. Ecol. Sociobiol. 51(6): 519-529 doi:10.1007/s00265-002-0473-3. 
Yamaguchi, H., Kikusui, T., Takeuchi, Y., Yoshimura, H., and Mori, Y. 2005. Social stress decreases marking behavior independently of testosterone in Mongolian gerbils. Horm. Behav. 47(5): 549-555, doi:10.1016/j.yhbeh.2004.12.009. PMID:15811356.

Zedrosser, A., Bellemain, E., Taberlet, P., and Swenson, J.E. 2007. Genetic estimates of annual reproductive success in male brown bears: the effects of body size, age, internal relatedness and population density. J. Anim. Ecol. 76(2): 368-375. doi:10.1111/j.1365-2656.2006.01203.x.

Zhang, J.X., Zhang, Z.B., and Wang, Z.W. 2001. Scent, social status, and reproductive condition in rat-like hamsters (Cricetulus triton). Physiol Behav. 74(4-5): 415-420. doi:10.1016/S0031-9384(01)00506-6. PMID:11790399. 


\section{Figure captions}

Fig. 1. Dorsal view of adult male brown bear (a), and magnification of border region (b) where the abundant sebum secretion was observed. Star indicates location of skin biopsy. Area of skin with observed secretion is limited to the midline between the first cervical vertebra and the second lumbar vertebra (dotted line). Border of abundant secretion is marked with arrow (visible sebum covering skin and hair) and arrowhead (dry skin and hair).

Fig. 2. Sebaceous glands in the back skin of a male brown bear and associated seasonal changes in size. Enlarged sebaceous glands were observed during the transitional (b) and breeding seasons (c). In pre- (a) and post- (d) breeding seasons, no enlarged sebaceous glands were observed. Arrow: enlarged sebaceous glands. Arrowhead: shriveled sebaceous glands. Scale bar $=1000 \mu \mathrm{m}$.

Fig. 3. Seasonal changes in indices of the sebaceous gland size in the back skin and plasma testosterone concentrations of intact bears. Indices tended to be higher during the transitional and breeding seasons than during the pre-breeding season $(P=0.05$ and $P=$ 0.07 , respectively), and were significantly higher than during post breeding season $(P<$ 0.05). Testosterone concentrations tended to be higher during the transitional, than during the pre-breeding season $(P=0.05)$, and significantly higher than during the post-breeding season $(P<0.05)$. Plasma testosterone concentrations during the breeding season were significantly higher than during pre- and post-breeding seasons $(P<0.05)$. Pre-breeding, $n$ $=4$; Transitional, $n=6$; Breeding, $n=12$; Post-breeding, $n=12$.

Fig. 4. Scatter plot of positively correlated sebaceous gland size indices and plasma testosterone concentrations in intact males $(r=0.78 ; P<0.05)$.

Fig. 5. Immunohistochemical staining for the androgen receptor (AR) in the sebaceous glands found in the back (a) and rump (b) skin samples from an intact male and in the back 
skin (c) sample of a castrated male, and negative control (d). Positive AR staining was observed in the nuclei of sebaceous gland cells in the back skin. Scale bar $=50 \mu \mathrm{m}$. These sections were photographed at a magnification $\times 400(\times 10$ ocular lens and $\times 40$ objective lens). 
Table 1. The number of animals examined during each sampling season.

\begin{tabular}{|c|c|c|c|c|c|c|c|}
\hline \multirow{2}{*}{ Facility } & \multirow{2}{*}{$\begin{array}{l}\text { Male } \\
\text { sexual } \\
\text { status }\end{array}$} & \multirow{2}{*}{$\begin{array}{c}\text { Pre-breeding } \\
\text { Season }\end{array}$} & \multirow{2}{*}{$\begin{array}{c}\begin{array}{c}\text { Transitional } \\
\text { season }\end{array} \\
\text { April }\end{array}$} & \multicolumn{2}{|c|}{$\begin{array}{c}\text { Breeding } \\
\text { season }\end{array}$} & \multicolumn{2}{|c|}{$\begin{array}{c}\text { Post-breeding } \\
\text { season }\end{array}$} \\
\hline & & & & May & June & August & October \\
\hline \multirow[t]{2}{*}{ A } & Intact & 4 & $2^{*}$ & 4 & 4 & 4 & 4 \\
\hline & Castrated & $\mathrm{NE}$ & 1 & $\mathrm{NE}$ & 1 & $\mathrm{NE}$ & 1 \\
\hline \multirow[t]{2}{*}{$\mathrm{B}$} & Intact & $\mathrm{NE}$ & 4 & $\mathrm{NE}$ & $4^{* *}$ & $\mathrm{NE}$ & 4 \\
\hline & Castrated & $\mathrm{NE}$ & 2 & $\mathrm{NE}$ & 2 & $\mathrm{NE}$ & 2 \\
\hline \multirow[t]{2}{*}{ Total } & Intact & 4 & 6 & \multicolumn{2}{|c|}{12} & \multicolumn{2}{|c|}{12} \\
\hline & Castrated & $\mathrm{NE}$ & 3 & \multicolumn{2}{|c|}{3} & \multicolumn{2}{|c|}{3} \\
\hline
\end{tabular}

NE: not examined

*: Only two of the four available males were examined.

**: The rump skin was collected from four intact bears in Facility B. 

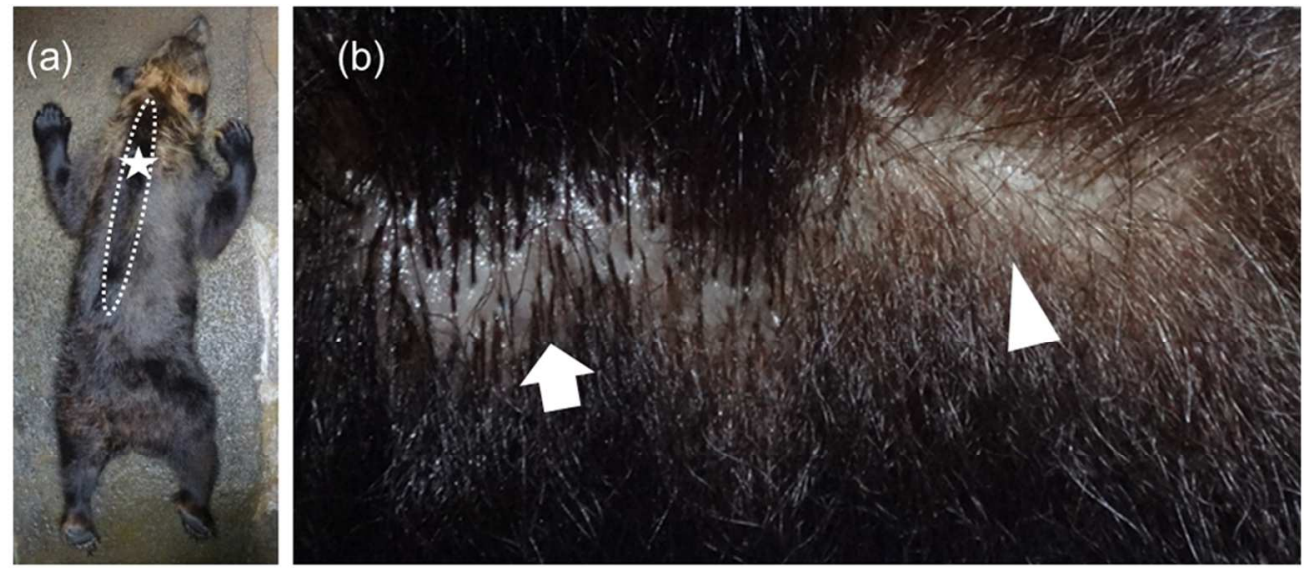

Fig. 1.

$182 \times 78 \mathrm{~mm}(300 \times 300 \mathrm{DPI})$ 

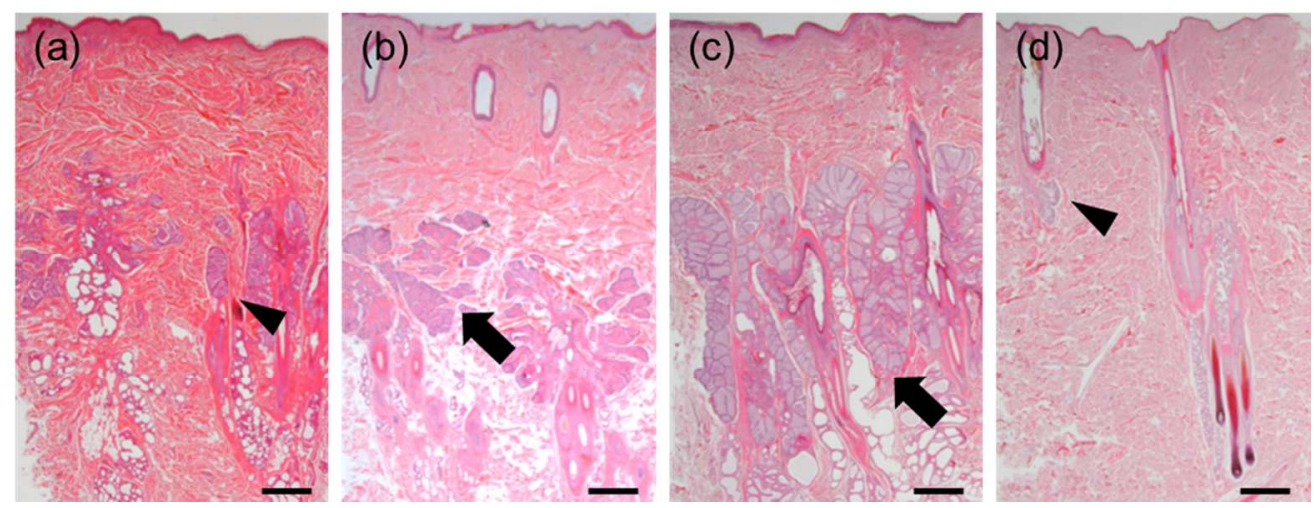

fig. 2 .

$181 \times 68 \mathrm{~mm}(300 \times 300$ DPI $)$ 

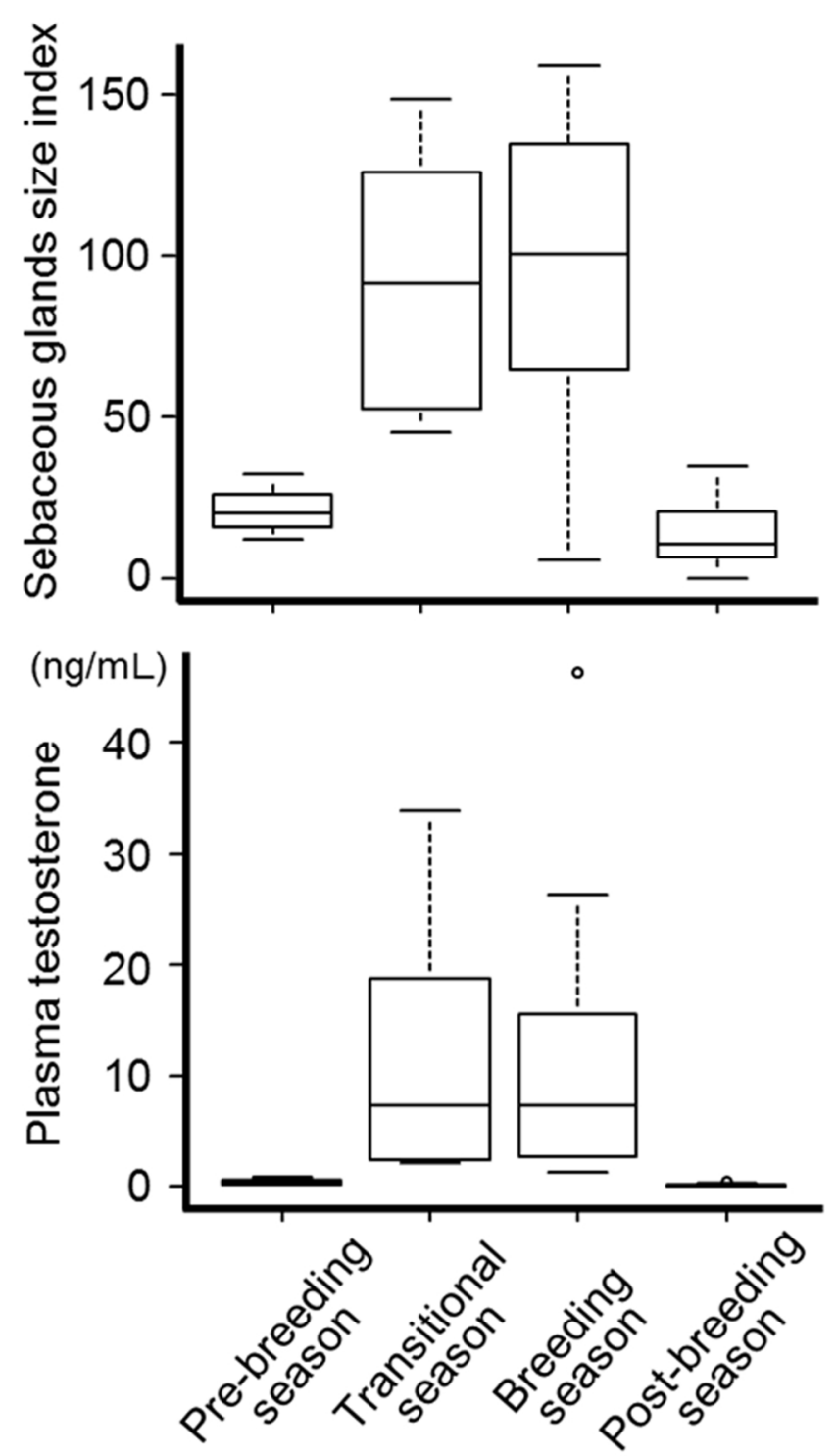

$86 \times 150 \mathrm{~mm}(300 \times 300$ DPI $)$ 


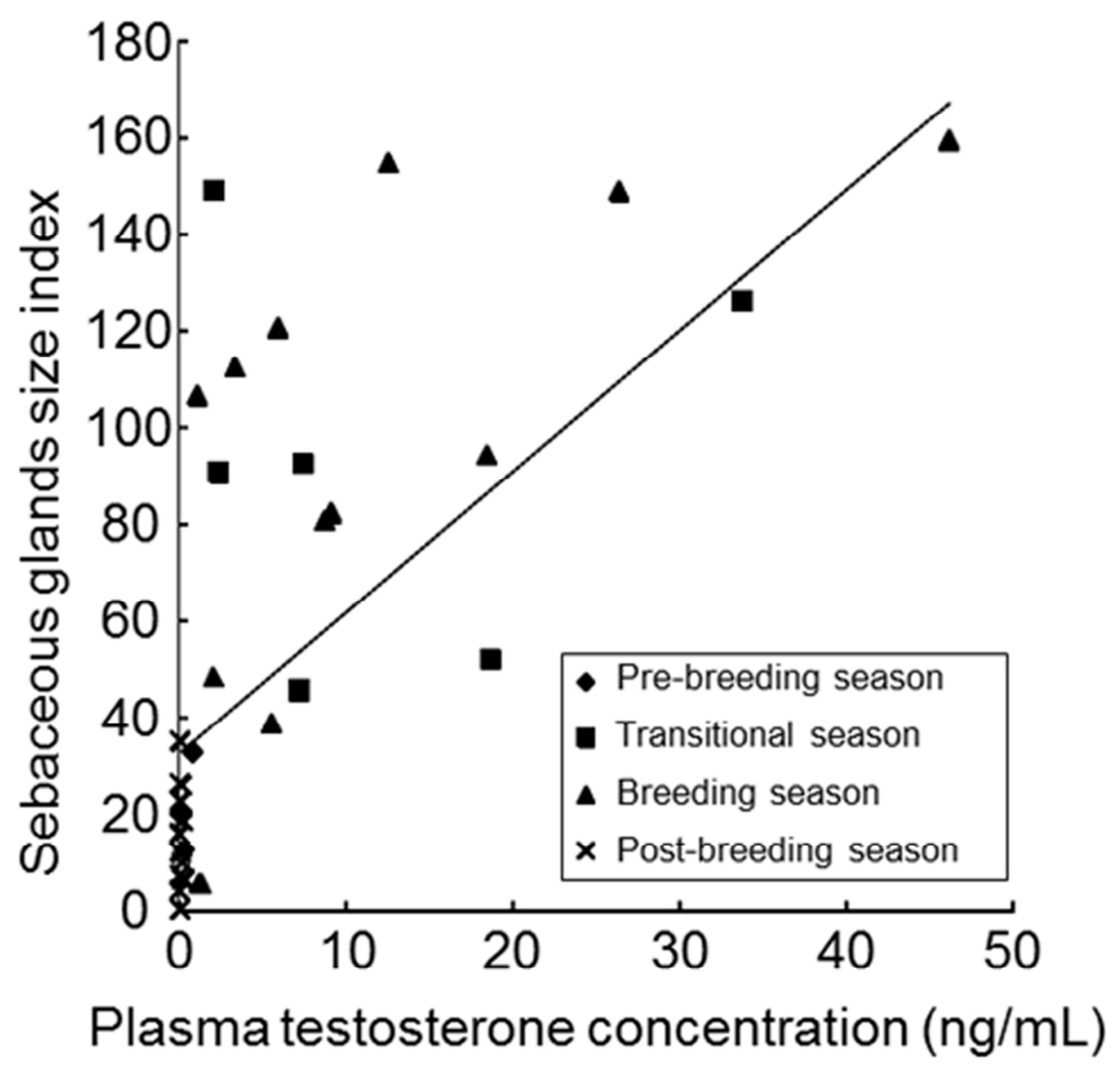

Fig. 4

$81 \times 77 \mathrm{~mm}(300 \times 300$ DPI $)$ 

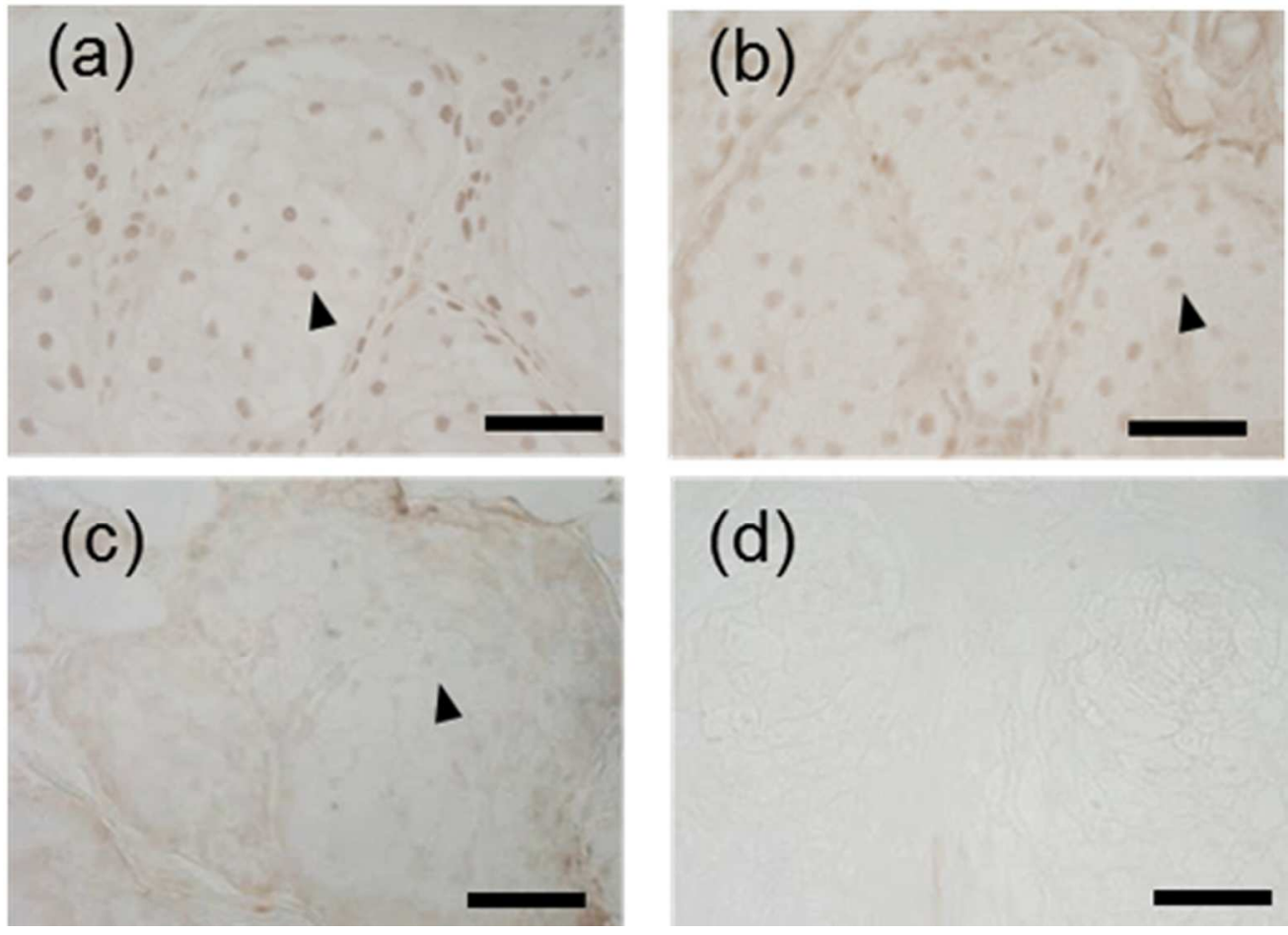

Fig. 5

$86 \times 62 \mathrm{~mm}(300 \times 300$ DPI $)$ 